

\section{Measurement of Employees on Human Resources with Fuzzy Logic}

\section{Zehra Demirel Ceren Çubukçu}

\section{Introduction}

Human resources have been greatly affected with the information technology revolution around the World, as in every field. The invention of the internet and the human resources beginning to benefit from this service created a compulsory competitive environment. The aim of human resources is to increase process efficiency by transforming their systems from traditional approaches to technological approaches in order to gain advantage in this competitive environment (LengnickHall \& Moritz, 2003).

Human resources management is a system that serves the interests and benefits of managers in organizations, and is designed to increase the performance of employees to the best level. Managers are faced with many situations that require them to make performance evaluations by using different data types while making decisions in their recruitment at the same time. The human resources department has many duties such as recruiting personnel, evaluating current employees' performance, as well as training and rewarding them. In short, human resources are the key to success or failure of an organization (Golec \& Kahya, 2007).

It is aimed to use artificial intelligence technologies, which are on the agenda of these recent times, in human resources management as in every field. There are too many definitions of artificial intelligence. We can define it with the following general statements: When certain data entries are made to the machines, the machines learn some information from these data entries. Using this information, they perform many higher cognitive functions such as decision making, reasoning, complex analytics, speech recognition, pattern recognition, and commenting on a situation. Machines imitate human intelligence and abilities with this technology. Topics covered are fuzzy logic, artificial neural networks, expert systems, natural language processing and genetic algorithms (Anderson, Rainie, \& Cohn, 2018).

Fuzzy logic, one of the artificial intelligence techniques, is a method in which intermediate values also exists instead of the classical logic with only 0 and 1 values based on ancient Greek philosophy. While classical logic includes definite results, approximate results are included in fuzzy logic. It aims to bring numerical expressions closer to human thoughts and is based on the set theorem. It offers more flexible results. Some uncertainties are encountered in decision making processes. Uncertainties can be made more apparent with fuzzy logic. Difficult and complex problems can be solved by using fuzzy logic approach in human resources management (Özdemir, Alaybeyoğlu, \& Balbal, 2019; Yildiz, 2006).

In human resources management, it is necessary to consider more than one parameter simultaneously in order to make an effective performance evaluation. Fuzzy logic can provide solutions to uncertainties by clustering with more than one parameter (Yager, 2002). The most valuable asset of an institution or organization is its employees. The employees of the institution greatly affect the level of success. Therefore, human resources management should focus on selecting people who are successful and talented in their jobs (Goes \& Limao, 2017).

The aim of this study is to measure and evaluate the performances at the stage of personnel evaluation and selection with the fuzzy sets theory and to draw the most accurate results for the benefit of the institution or organization accordingly. In this study, fuzzy logic based Mamdani model will be used as a solution method. In this system, 9 rule bases have been applied. The centers of gravity were determined by defining min-max values and then, the defuzzification processes were carried out (Chan-ohMin, Dae-wooLee, Kyeum-raeCho, Sung-jinJo, Jang-sikYang, \& WonbooLeec, 2011). The level of success is measured both according to their experience and graduation level as being a bachelor's, master's or doctoral degree graduate, being - inexperienced, 2-year experienced or 10-year experienced The proposed Mamdani model will be tested using MATLAB.

\section{Literature Review}

A new method based on the fuzzy logic principle has been proposed according to human resources management for the employees in the hotel industry in order to measure the performance and select the most suitable and useful employee. This method has introduced a scoring system in human resources management to reach a correct approach. Human resources used Mamdani fuzzy logic for measuring the performance. The course of the study was as follows: A group of experts have been included in the system and the rules have been established. Then, a survey was conducted with human resources managers. Afterwards, everyone's human resources performance scores have been calculated. For performance measurement in medium-sized companies, where there are membership rules, functions and domains that can be used a template have been created. The performance measurement result of the human resources management resulted in a clear number of degree 3 . According to this, all values above the 3rd degree mean a high performance, the 3rd degree means an average performance and those below the 3rd degree means a low performance indicator (Ruzic, Skenderovic, \& Lesic, 2016).

Enterprise resource planning (ERP) is a software program that supports many basic business processes such as production, finance, sales, marketing and human resources. It is very important to choose an ERP that suits the needs of the organization. A method based on fuzzy logic is suggested to select the right ERP solution. Several criteria have been used to select the appropriate ERP. These are functionality, cost, and vendor support. Using the membership function for each of these criteria, the Mamdani inference system has been 
developed. The created model has 3 inputs and 1 output. The main purpose of this model is to measure the different ERP systems. For this, 96 fuzzy rule bases were designed using the Mamdani method. The rules created here are based on expert knowledge and experience in the field. For example, if functionality $=0.573$, cost $=$ 0.596 , and vendor support $=0.5$ were evaluated as input, the output value has resulted in 0.692 , indicating that the output result was a high value (Vahidi, Salookolayi, \& Yavari, 2014).

The biggest concern in human resources management is the huge damage it will cause to the organization, if the right person is not recruited. For this reason, using fuzzy logic in human resources management has been valuable. In order to gain an advantage in today's competitive environment, information technology applications help managers to make decisions significantly. Decision support systems are a sub-branch of computer-based information systems that support decision making. An accurate decision support system is a system that makes decisions by interpreting the information extracted from raw data, personal data and existing business models. In normal life, it is difficult to think about many features at once and make the right decision, and because of this difficulty, these decisions seem to take place in a blurry environment. Fuzzy inference maps an output by formulating input data using fuzzy logic. Fuzzy logic has two types of inference systems; Mamdani and Sugeno. Both show different results in the final output (Talaei, 2012).

Since human resources are considered as the most important source of the competitive business environment today, choosing the right person has become more important than ever. While making a decision, human resources follow the method process in an empirical practice with two known inference modelings such as Mamdani and Sugeno in a fuzzy environment. As in many real problems in the world, personnel selection in human resources has been solved with fuzzy logic (Golec \& Kahya, 2007). A study attempts to find the most suitable candidate for a machine maintenance manager position at a selected organization including inconclusive judgments with Mamdani and Sugeno techniques. . The effectiveness of the proposed implications was tested with MATLAB / simulink program (Tinkir, Doganalp, \& Doganalp, 2015).

Liang and Wang (1994) used fuzzy logic, which is a multi-criteria decision-making mechanism (MCDM), for personnel selection. The MCDM procedure should include quantitative and qualitative data for staff selection. The decision approach should take into account language variables or fuzzy data as well as clear data. Human resources managers in an organization should consider many factors such as personality, leadership and communication skills when selecting personnel. Fuzzy set theory is an effective tool, which also includes uncertain judgments in the personnel selection process. Solutions have been presented to select the most suitable person among the pool of candidates. An institution conducts interviews, skill and achievement tests to measure the performance potential of applicants (Wang \& Yen, 1999). It is very important to evaluate the performance of the employees in order to increase the quality of the work. It provides the basis for both motivation and identification as well as improvement of weaknesses. As a result of the performance evaluations of the employees in an organization, management can observe their weaknesses and strengths. Fuzzy approach for performance evaluation has introduced a new supervisory system. In this system, all settings of the user interfaces have been developed by protecting the data with the MS Access database (Shaout \& Yousif, 2014).

Two fuzzy clustering approaches can be preferred in performance evaluations. The first is the traditional fuzzy method that uses rules to evaluate overall scores from many linguistic fuzzy inputs without any intermediate fuzzy logic. Traditional methods generate too many rules and it is difficult for experts to consider all aspects and formulate rules with proper weights. For these reasons, the process becomes more difficult and complex. The second approach explains the relationship between performance-critical details and identifies new large groups accordingly. This approach, also known as fuzzy reasoning, is the stage where it is possible and flexible to give different weights (Shaout \& Trivedi, 2013).

\section{Materials and Methodology}

Fuzzy logic has two types of inference systems named Mamdani and Sugeno. The biggest difference between the two is that the value is extracted after some kind of defuzzification process. In the Sugeno defuzzification process, the result is obtained in the form of a function. Mamdani inference method was preferred in this study. Results can be produced with the other method as well. The reason why Mamdani method is preferred is that the membership levels incoming from the fuzzy unit are wanted to be associated with the output membership values by using the minimum relationship operator. The purpose of the study is a system that decides whether the person applying to human resources is suitable for that job posting. According to the experience and graduation degree of the applicant, it is asked to decide whether to recruit or not by measuring the experience of the applicant as inexperienced, 2-yearexperienced or 10-year-experienced in addition to measuring the graduation degree as associate, master's or doctorate.

Depending on these, the results are asked to be evaluated as less successful, moderately successful and very successful. Fuzzification process is performed depending on the input functions, then inference is obtained according to the determined rule base, finally the output is reached by defuzzification.

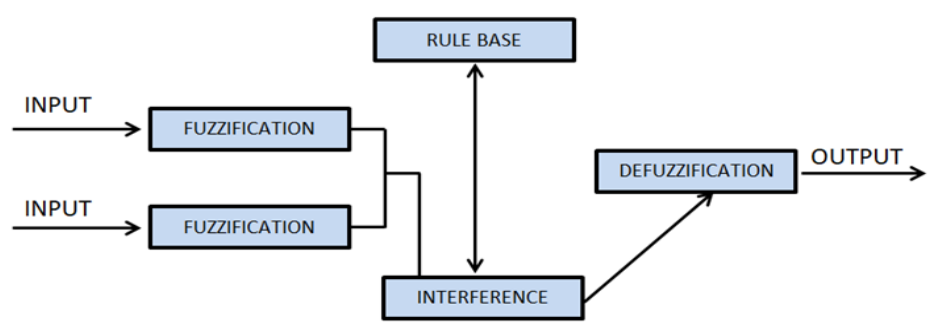

Figure 1. Components of a fuzzy logic controller

Source: Authors' own compilation

The rules determined according to the wishes of the working managers in the institution are shown below: 
New Graduated: NG Associate Degree: AD

2-Year Experienced: 2YE Master's Degree: MD

10-Year Experienced: 10YE PhD Graduate: PhD

Less Successful: LS

Medium Successful: MS

\section{Table 1: Rule Base}

Very
If (inputl is NG) and (input2 is AD ) then (outputl is LS ) (1)
If (inputl is NG) and (input2 is MD ) then (outputl is MS ) (1)
If (inputl is NG) and (input2 is PhD ) then (outputl is MS ) (1)
If (inputl is 2YE) and (input2 is AD) then (outputl is MS ) (1)
If (inputl is 2YE) and (input2 is MD ) then (outputl is MS ) (1)
If (inputl is 2YE) and (input2 is $\mathrm{PhD}$ ) then (outputl is MS ) (1)
If (inputl is 10YE) and (input2 is AD ) then (outputl is MS ) (1)
If (inputl is 10YE) and (input2 is MD ) then (outputl is VS ) (1)
If (inputl is 10YE) and (input2 is $\mathrm{PhD)} \mathrm{then} \mathrm{(outputl} \mathrm{is} \mathrm{VS} \mathrm{)} \mathrm{(1)}$

\section{Source: MATLAB Output}

When the person submits the pre-application form to the institution, a scoring is made according to their work experience and graduation status. These scores are entered into the system as input values. Then; a result is obtained according to the center of gravity after performing min-max operation with the Mamdani method.

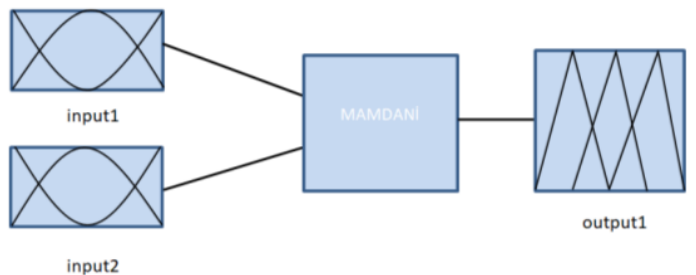

Figure 2. Mamdani Fuzzy System

Source: MATLAB Output

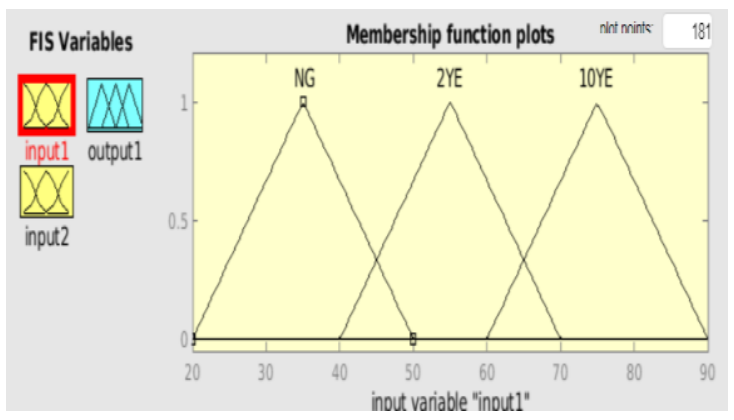

Figure 3. Input 1 Variables

Source: MATLAB Output

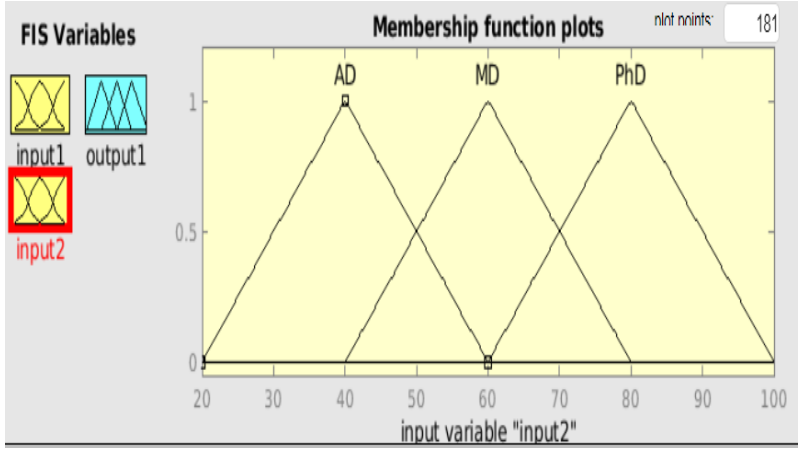

Figure 4. Input 2 Variables

Source: MATLAB Output

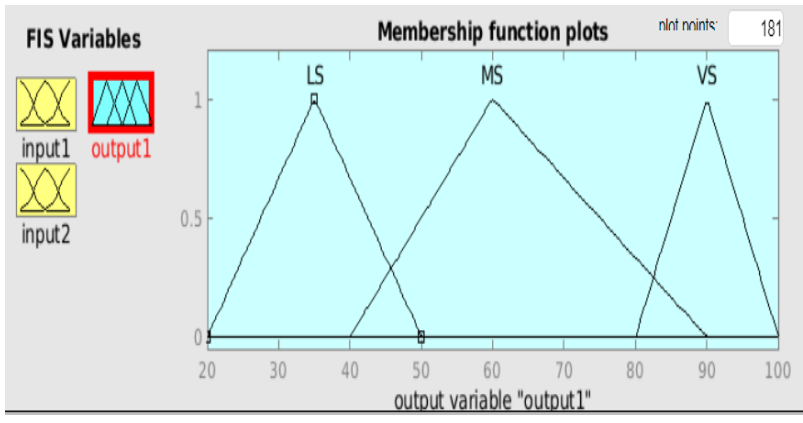

Figure 5. Output 1 Variables

Source: MATLAB Output

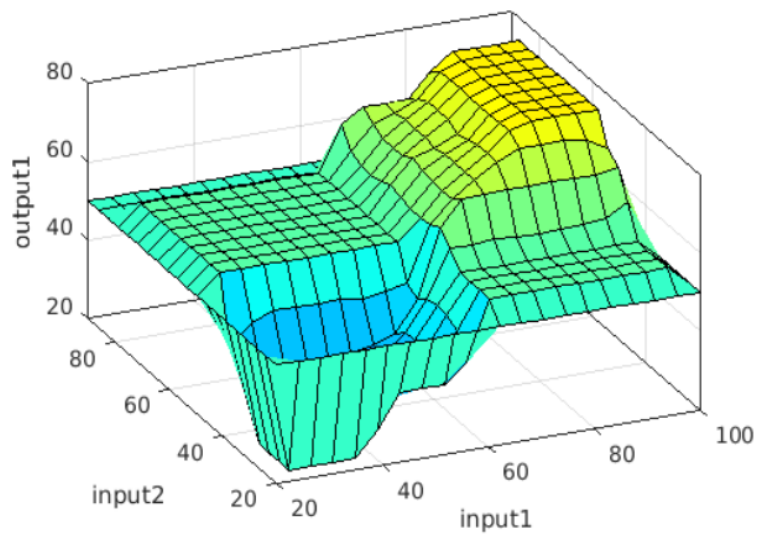

Figure 6. Matlab Surface

Source: MATLAB Output
NG: $25 \mathrm{p}$.
2YE: $60 \mathrm{p}$.
AD: $25 \mathrm{p}$.
10YE: $80 \mathrm{p}$.
Ph.D. G:90 p.

If the applicant is an inexperienced and an associate degree graduate, a less successful result is obtained by performing a min-max operation according to the center of gravity in the joint evaluation. 


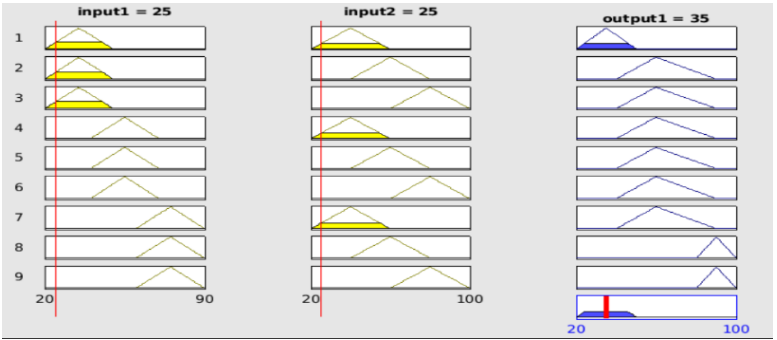

Figure 7. New Graduated and Associate Degree

\section{Source: MATLAB Output}

If the applicant is an inexperienced and a Master's degree graduate, a medium successful result is obtained by performing a min-max operation according to the center of gravity in the joint evaluation.
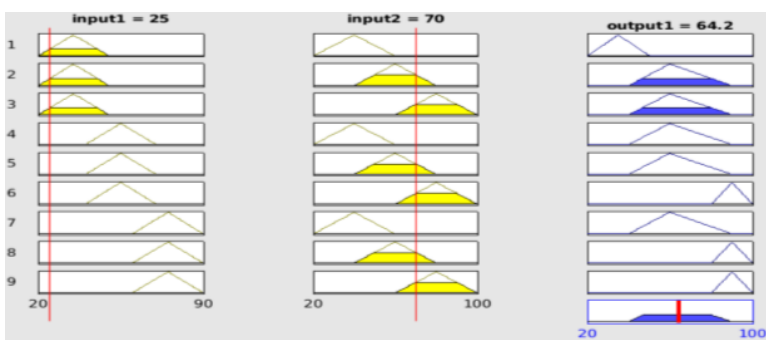

Figure 8. New Graduated and Master's Degree

\section{Source: MATLAB Output}

If the applicant is inexperienced and a $\mathrm{Ph} . \mathrm{D}$. graduate, a medium successful result is obtained by performing a min-max operation according to the center of gravity in the joint evaluation.

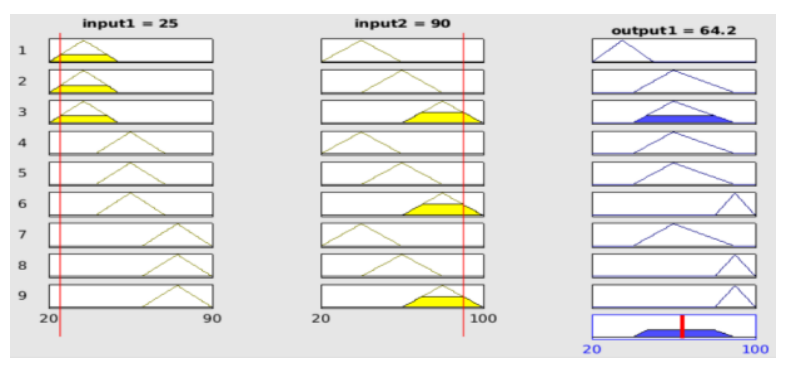

Figure 9. New Graduated and Ph.D. Graduate

Source: MATLAB Output

If the applicant is a 2-year experienced and an associate degree graduate, a medium successful result is obtained by performing a min-max operation according to the center of gravity in the joint evaluation.

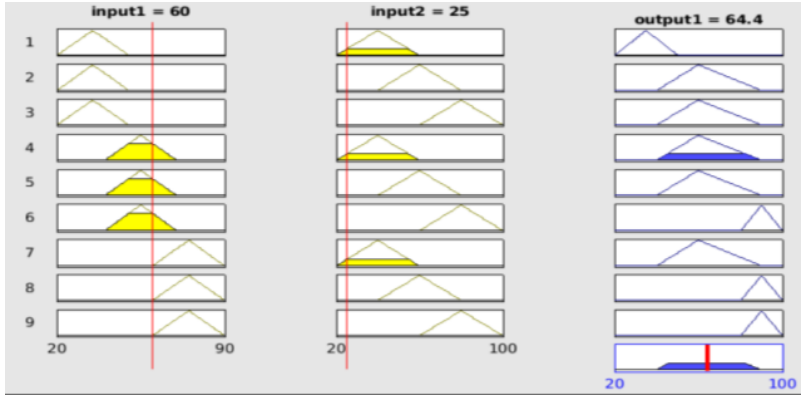

Figure 10. 2-Year Experienced and Associate Degree

Source: MATLAB Output

If the applicant has 2 years of experience and have a master's degree, a medium successful result is obtained by performing a min-max operation according to the center of gravity in the joint evaluation.

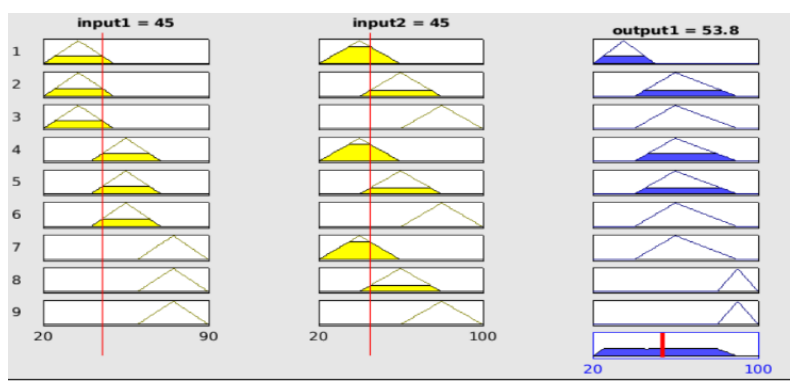

Figure 11. 2-Year Experienced and Master's Degree

Source: MATLAB Output

If the applicant has 2 years of experience and have a doctorate degree, a very successful result is obtained by performing a min-max operation according to the center of gravity in the joint evaluation.
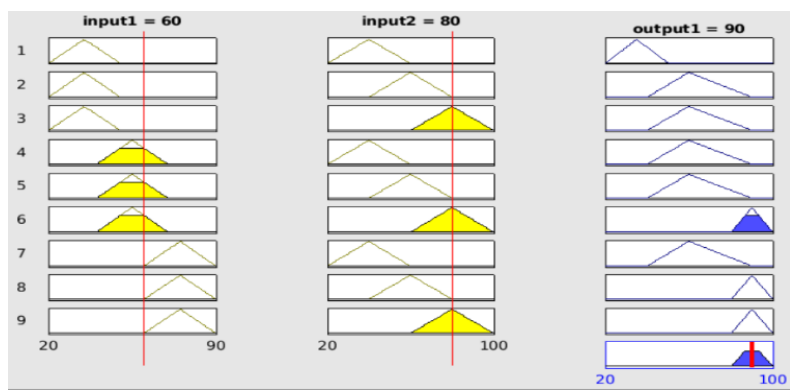

Figure 12. 2-Year Experienced and Ph.D. Graduate

Source: MATLAB Output

If the applicant is 10 years experienced and an associate's degree graduate, a medium successful result is obtained by performing a min-max operation according to the center of gravity in the joint evaluation. 


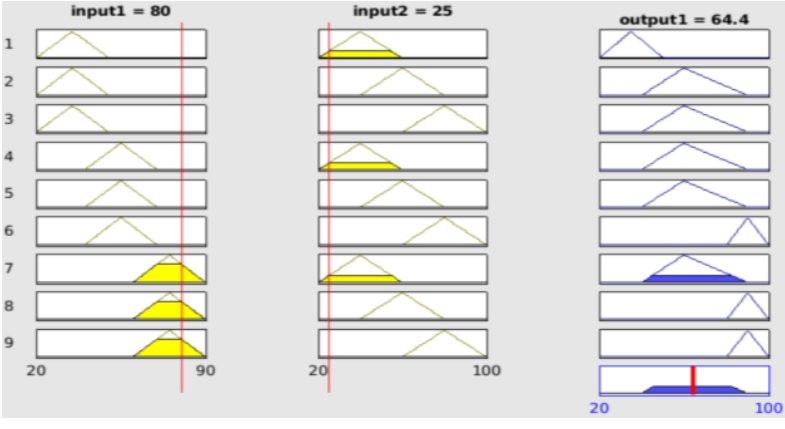

Figure 13. 10-Year Experienced and Associate Degree

Source: MATLAB Output

If the applicant is 10 years experienced and a Master's degree graduate, a very successful result is obtained by performing the min-max process according to the center of gravity in the joint evaluation.
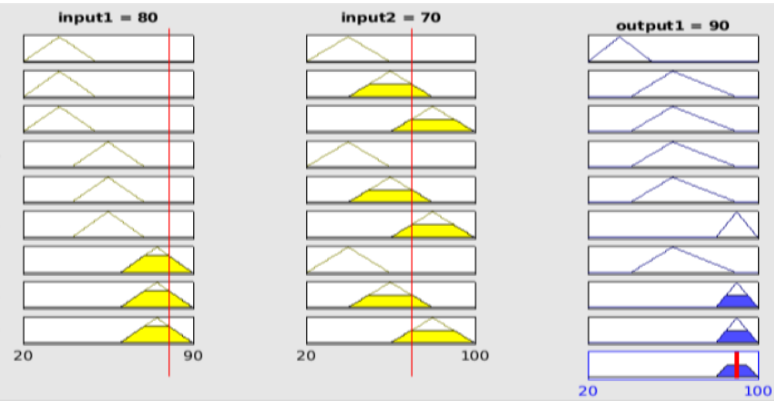

Figure 14. 10-Year Experienced and Master's Degree

\section{Source: MATLAB Output}

If the applicant is 10 years experienced and a $\mathrm{Ph} . \mathrm{D}$. graduate, a very successful result is obtained by performing the min-max process according to the center of gravity in the joint evaluation.

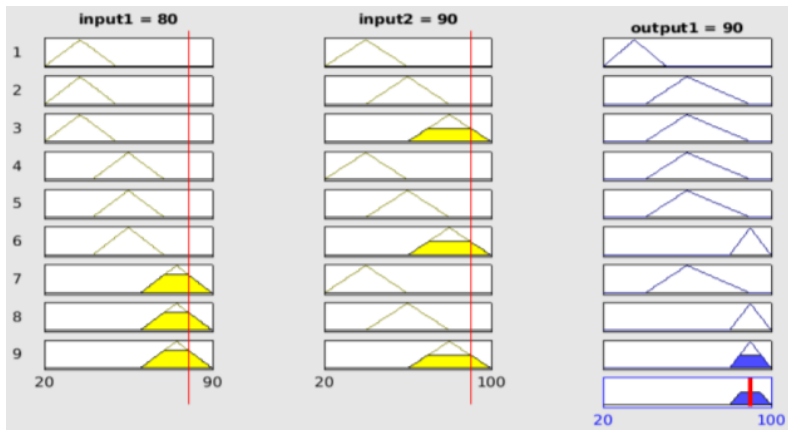

Figure 15. 10-Year Experienced and Master's Degree Ph.D. Graduate

Source: MATLAB Output

\section{Conclusion}

The human resources management system is the information backbone of an organization and reaches all departments within a business. The most valuable asset of an institution is human beings. For this reason, expensive investments are made in human resources. It is very important to choose appropriate personnel for the needs of the institution. In this study, a Mamdani method is presented to select the appropriate element for the requirements based on fuzzy logic inference. Fuzzy logic decision making with Mamdani inference models was performed using the MATLAB / simulink ready toolbox.

This model has 2 inputs and 1 output. Triangular membership functions are used for each entry criterion. The verbal criteria given by the managers in the organization have been transformed into numbers by using the triangle membership functions. Results have been drawn after the clarification process of these numbers according to their membership degrees. According to the results of the triangle membership, a ranking was made and it was determined whether there was a suitable candidate for the personnel needs. Very successful, moderately successful and less successful people are ranked among themselves according to the result of the membership coefficient. In this organization, the first 5 people were chosen among those who were very successful as a suitable candidate. For people with medium achievement results, other positions were registered in the system to be considered. Less successful people are not considered for positions in the company. This study shows that human resources are suitable for multi-criteria group decision making with Mamdani method using fuzzy logic models in eligible candidate selection processes.

Adaptive network-based fuzzy inference system (ANFIS) can be used for human resources personnel selection in future studies. ANFIS is a type of artificial neural network method based on Takagi-Sugeno fuzzy inference system. ANFIS uses both neural networks and fuzzy logic inference methods in an integrated manner. This method can be used for future studies as it benefits from both of the structures. More effective decisions can be made in human resource selection processes with fuzzy logic inferences.

\section{References}

Anderson, J., Rainie, L., \& Cohn, S. (2018). Artificial Intelligence and the Future of Humans. pewresearch, 50-62.

Chan-ohMin, Dae-wooLee, Kyeum-raeCho, Sung-jinJo, Jang-sikYang, \& Won-booLeec. (2011). Control of approach and landing phase for reentry vehicle using fuzzy logic . sciencedirect, 269-282.

Goes, A. S., \& Limao, R. C. (2017). Fuzzy System for Human Resource Performance Evaluation. Las Vegas, NV, USA: IEEE.

Golec, A., \& Kahya, E. (2007). A fuzzy model for competency-based employee evaluation and selection. ScienceDirect, 143-161.

Lengnick-Hall, M. L., \& Moritz, S. (2003). The impact of e-HR on the human resource management function. Journal of Labor Research , 24, pages365-379. 
Özdemir, A., Alaybeyoğlu, A., \& Balbal, K. F. (2019). Bulanık Mantı̆̆ın Eğitim Alanındaki Uygulamalar1. dergipark, 45-50.

Ruzic, M. D., Skenderovic, J., \& Lesic, K. T. (2016). Application of the Mamdani Fuzzy Inference Ssystem to Measuring HRM Performance in Hotel Companies - A Pilot Study. researchgate, 53-65.

Shaout, A., \& Trivedi, J. (2013). Performance Appraisal System - Using a Multistage Fuzzy Architecture. International Journal of Computer and Information Technology, III(2).

Shaout, A., \& Yousif, M. K. (2014). Employee Performance Appraisal System. International Journal of Computer Science \& Information Technology (IJCSIT), IV(6).

Talaei, G. (2012). Using Fuzzy Decision Support Systems in Human Resource Management. Journal of Basic and Applied, 2035-2039.

Tinkir, M., Doganalp, B., \& Doganalp, S. (2015). Human Resource Selection Process by Using Various Fuzzy Logic Techniques. Proceedings of the Workshop on Foundations of Informatics, 24-29.

Vahidi, J., Salookolayi, D. D., \& Yavari, A. (2014). A Model for Selecting an Erp System with Triangular Fuzzy Numbers and Mamdani Inference. Journal of Mathematics and Computer Science, 46-54.

Wang, L., \& Yen, J. (1999). Extracting fuzzy rules for system modeling using a hybrid of genetic algorithms and Kalman filter. Science Direct, 353-362.

Yager, R. (2002). Fuzzy Optimization and Decision Making. sciencedirect, 13-41.

Yıldız, M. (2006). Bilişim Teknolojilerinin Stratejik Rekabeti. Selçuk Üniversitesi, Konya. 